\title{
Seguridad alimentaria y transformación agraria sostenible
}

\author{
Yoel López Gamboa \\ https://orcid.org/0000-0002-9596-443X \\ yoel111975@gmail.com \\ Ciudad de Guayaquil. Provincia Guayas. Ecuador \\ Universidad Metropolitana de Ecuador. \\ Marcos Elpidio Pérez \\ https://orcid.org/0000-0002-8040-283X \\ Ciudad de Guayaquil. Provincia Guayas. Ecuador \\ Universidad Metropolitana de Ecuador.
}

\section{RESUMEN}

La seguridad alimentaria parte de la perspectiva de que cada persona disponga de alimentación básica en cantidad y calidad acorde a sus necesidades nutricionales, proceso que se ve afectado severamente por causas multifactoriales. El objetivo del artículo es contextualizar la influencia de la seguridad alimentaria en la nutrición y el estado de salud. Se realizó una investigación documental, mediante revisión en las bases de datos; Google académico, Scielo, PubMed, Medline y Dialnet, decantándose 52 artículos de los cuales se tomaron en consideración 31 por ajustarse en mayor grado al tema de análisis. Entre los resultados: La inseguridad alimentaria constituye un serio problema para el mantenimiento de un estado de salud óptimo. La falta de acceso a los alimentos en cantidad y calidad adecuada afecta fundamentalmente a la población de países en desarrollo, dentro de ellos existen grupos poblacionales más vulnerables dentro de los que destacan; los niños, mujeres embarazadas, población indígena y personas de bajos ingresos económicos. Urge una atención diferenciada a los programas de alimentación por parte de los gobiernos, que sean sostenibles y de acceso universal.

Pablas claves: seguridad alimentaria; seguridad nutricional; agricultura ecológica 


\title{
Food security and sustainable agricultural transformation
}

\begin{abstract}
Food security starts from the perspective that each person has basic food in quantity and quality according to their nutritional needs, a process that is severely affected by multifactorial causes. The aim of the article is to contextualize the influence of food security on nutrition and health status. A documentary investigation was carried out, by reviewing the databases; Academic Google, Scielo, PubMed, Medline and Dialnet, choosing 52 articles of which 31 were taken into consideration because they adjusted to a greater degree to the subject of analysis. Among the results: Food insecurity constitutes a serious problem for the maintenance of an optimal state of health. The lack of access to food in adequate quantity and quality fundamentally affects the population of developing countries, within them there are more vulnerable population groups, among which they stand out; children, pregnant women, indigenous population and people with low income. There is an urgent need for differentiated attention to food programs by governments, which are sustainable and of universal access.
\end{abstract}

Key words: food security; nutritional security; ecological agriculture

Artículo recibido: 20 diciembre. 2021 Aceptado para publicación: 10 enero 2022 Correspondencia: yoel111975@gmail.com Conflictos de Interés: Ninguna que declarar 


\section{INTRODUCCIÓN}

La seguridad alimentaria parte de la perspectiva de que cada persona disponga de alimentación básica en cantidad y calidad acorde a sus necesidades nutricionales, proceso que se ve afectado severamente por diferentes causas, dentro de las cuales se pueden citar la minería, la explotación petrolera, la falta de recursos económicos, entre otras. (Borrego \& Enrique, 2020). El cambio climático es otro factor que coadyuba a la existencia de inseguridad alimentaria en lo que hoy viven millones de personas en el mundo, sobre todo la población más vulnerable, dentro de los que destacan; los niños, mujeres embarazadas, población indígena y personas de bajos ingresos económicos. (Altier et al., 2020)

Desde los años setenta que se define por primera vez el termino de seguridad alimentaria, el concepto ha ido evolucionando con el paso del tiempo, agregando otras dimensiones; no solo la disponibilidad y la producción alimentaria son contenidas en el mismo, ahora comprende la disponibilidad económica y física, además de incluir en tiempos recientes las preferencias culturales y la inocuidad, llegando a considerar desde el año 1996 como un derecho humano, proclamado en la Cumbre Mundial de alimentación celebrada por la Organización de las Naciones Unidas para la Alimentación y la Agricultura (FAO). (Aguilar-Estrada et al., 2019)

Un factor que puede afectar considerablemente la calidad de los alimentos es el uso de sustancias de naturaleza hormonal tanto en la ganadería como en la acuicultura, así como el uso de antibióticos. En la región asiática, y más específicamente en China se usa de una manera desmedida los antibióticos en la acuicultura, lo que cobra una vital importancia al ser este país el de mayor acuicultura a nivel mundial; los antibióticos pueden ser causa de enfermedades para las personas que consumen alimentos que han sido inoculado con dichas sustancias, se pueden desencadenar reacciones alérgicas, además de contribuir a la resistencia microbiana, siendo este último fenómeno motivo de preocupación de la Organización Mundial de Salud (OMS) por su crecimiento exponencial en los últimos años. (Liu et al., 2017)

El uso de sustancias genéticamente modificadas para la alimentación animal que posteriormente sirven de alimentos a la población humana constituye otro punto de preocupación para la comunidad internacional; sobre este punto no existe un consenso claro en la comunidad científica, incluyendo la OMS. Es un objetivo pendiente de la Organización de las Naciones Unidas para la Agricultura y la Alimentación (FAO) 
evaluar con profundidad los efectos que ejercen para la salud humana el uso de piensos modificados genéticamente en la alimentación ganadera, pues existe mucha especulación en este sentido. (Fernandez \& Paoletti, 2018)

Con el objetivo de contextualizar la influencia de la seguridad alimentaria en la nutrición y el estado de salud se realizó el presente trabajo.

\section{MATERIALES Y MÉTODOS}

Se realizó una investigación documental, mediante revisión en las bases de datos; Google académico, Scielo, PubMed, Medline y Dialnet, decantándose 52 artículos de los cuales se tomaron en consideración 31, de los últimos 5 años, por ajustarse en mayor grado al tema de análisis. La revisión se realizó en los idiomas español, inglés y portugués con los siguientes términos de búsqueda: seguridad alimentaria, seguridad nutricional y agricultura ecológica.

\section{RESULTADOS Y DISCUSIÓN}

En cuanto a la agricultura se conoce que el uso de fertilizantes químico como estrategia para lograr un incremento de los rendimientos en los cultivos, no solo es una fuente de contaminación ambiental, sino que constituye un serio problema que amenaza la salud humana al ser una de las causas que hoy se conocen que pueden desencadenar diferentes enfermedades, siendo las cancerosas las más notables. Para lograr una agricultura sostenible en armonía con el medio ambiente y minimizar los daños a la salud se deberán seguir otras estrategias, como el uso de biofertilizantes e introducir tecnología novedosa como la nanotecnología. (Ramos-Ulate et al., 2021)

La nanotecnología garantiza con elevada precisión la incorporación de diferentes nutrientes a los fertilizantes que posteriormente serán incorporados a los cultivos que alimentarán a los seres humanos, además de lograr armonía con el medio ambiente, elemento que destaca en la etapa actual donde el cambio climático está provocando modificaciones significativas en la composición nutricional de los diferentes cultivos alimentarios, generando inseguridad en cuanto a cantidad y calidad de nutrientes en los alimentos de origen vegetal. (Borrego \& Enrique, 2020), (Ramos-Ulate et al., 2021).

Los productos alimentarios de origen vegetal constituyen un grupo importante de alimentos, pues del mundo vegetal provienen tanto macronutrientes (proteínas, carbohidratos y proteínas) como micronutrientes (vitaminas y minerales), los cuales en las últimas décadas han sido afectados en cuanto a cantidad y calidad por diferentes 
causas, siendo las más destacadas el uso de sustancias químicas y el cambio climático que ha afectado la calidad del suelo y consiguientemente los nutrientes de los alimentos. (Rumiato \& Monteiro, 2017)

Los contaminantes que se han descrito en la bibliografía científica actual destacan la presencia de metales pesados, herbicida e insecticida, los cuales se conoce que alteran el equilibrio del organismo humano provocando diferentes enfermedades. Es muy conocido el caso de la contaminación con cadmio y su relación con enfermedades neurológicas. (Rumiato \& Monteiro, 2017). Dentro de los herbicidas e insecticidas los que más daño se sabe provocan en la salud son los organoclorados y los organofosforados, siendo este último asociado en varios estudios con enfermedades cancerosas como linfoma no Hodgkin, mieloma múltiple y leucemias. (Moura et al., 2020)

El herbicida más utilizado a nivel mundial, con el objetivo de lograr un incremento en el rendimiento de cultivos agrícolas es el Glifosato, sobre todo en los cultivos de maíz, soja y frijol, constituyendo éstos alimentos la base alimentaria de muchas regiones, específicamente en población de bajos ingresos económicos; se han identificado en dichos alimentos residuos del insecticida, los cuales han sido asociados en diferentes investigaciones con severos daños a la salud en distintos órganos y sistemas del organismo humano, como son: alteraciones en la microbiota intestinal, daño en el sistema renal, disrupción del sistema endocrino, y afectaciones en el sistema neurológico entre otras. Dentro de las enfermedades descrita con asociación al Glifosato se pueden mencionar, alteraciones metabólicas, estrés oxidativo, enfermedades carenciales por déficit de manganeso y varios tipos de cáncer. (Tofiño Rivera et al., 2020).

Los efectos del cambio climático sobre la estabilidad de la naturaleza son notables, resaltando los desastres naturales (intensas lluvias, intensas sequías, incremento de la emanación de dióxido de carbono, etc.), mismos que impactan directamente en la inestabilidad de cultivos con fines alimentarios, tanto en cantidad como en su composición nutricional. Se ha evidenciado que las cantidades de nutrientes en determinados alimentos han disminuidos con respecto a etapas anteriores. (Borrego \& Enrique, 2020) Además de los factores anteriormente mencionados existen otros no menos importantes que también afectan la seguridad alimentaria derivados del cambio climático, como se muestra en la siguiente tabla.

Tabla. Vínculos entre cambio climático y seguridad alimentaria 


\begin{tabular}{|l|l|}
\hline \multicolumn{1}{|c|}{ Efectos del cambio climático } & \multicolumn{1}{c|}{$\begin{array}{c}\text { Consecuencias sobre la seguridad } \\
\text { alimentaria }\end{array}$} \\
\hline $\begin{array}{l}\text { Aumento de las temperaturas, cambios en } \\
\text { las precipitaciones (inundaciones). }\end{array}$ & $\begin{array}{l}\text { Variaciones en las plagas y } \\
\text { enfermedades de plantas y animales. }\end{array}$ \\
\hline $\begin{array}{l}\text { Afectación en el procesamiento y envasado } \\
\text { de alimentos. }\end{array}$ & $\begin{array}{l}\text { Aumento de las pérdidas principalmente } \\
\text { en agricultores de pequeña escala. }\end{array}$ \\
\hline $\begin{array}{l}\text { Afectación en la distribución y venta } \\
\text { dependientes de infraestructuras. }\end{array}$ & $\begin{array}{l}\text { Cierre de puertos marítimos, aéreos y } \\
\text { daño de carreteras. }\end{array}$ \\
\hline $\begin{array}{l}\text { Afectación de los consumidores. } \\
\text { Aumentos de los precios de los } \\
\text { alimentos. }\end{array}$ \\
\hline
\end{tabular}

Fuente: (Borrego \& Enrique, 2020)

Referente a los alimentos de origen animal la situación es similar a los de origen vegetal, de manera general las amenazas provienen del mismo origen. En las últimas décadas la creciente demanda de alimentos ha conducido a que la alimentación animal esté basada en piensos fabricado con alimentos vegetales obtenidos por modificación genética, tal como el maíz y la soya, lo que ha conducido a modificaciones en la calidad de los nutrientes de dichos animales y con ello la aparición de determinadas enfermedades asociadas a los citados cambios. (Pérez-Tamayo et al., 2020)

Una de las causas que ha modificado considerablemente la salud humana y a que su vez ha sido consecuencia del impacto del hombre sobre el medio ambiente está relacionado con la disbiosis intestinal, que ha sufrido directamente el impacto del cambio climático, y de la dieta; pues al modificarse los microorganismos que normalmente se introducen al organismo humano proveniente de los alimentos tanto de origen animal como vegetal, se modifica el microbiana intestinal humano generando serios problemas de salud donde interviene directa o indirectamente la flora intestinal (Weiss \& Hennet, 2017), (Thursby \& Juge, 2017)

Las investigaciones actuales apuntan a que el microbiota intestinal no solo participa en el mantenimiento del estado de salud intestinal, sino que a través de diferentes mecanismos de interacción afecta el estado de salud de los demás órganos del cuerpo humano destacando el sistema nervioso central, el sistema endocrino, sistema inmunológico y la piel. (Ni et al., 2017), (Castañeda Guillot, 2018).Anteriormente se pensaba que el útero de la madre era totalmente estéril, pero se sabe actualmente que también se encuentra colonizado por diferentes gérmenes que desde ese momento pueden condicionar el estado 
de salud del futuro individuo, además de provocar algunas enfermedades de la madre durante el embarazo, tal como la hipertensión arterial. (Butel et al., 2018), (Baldassarre et al., 2019)

El desequilibrio del microbiota intestinal se ha asociado con múltiples procesos patológicos que afectan prácticamente a todo el organismo humano, pudiendo citar las siguientes: Síndrome metabólico (Hipertensión arterial, diabetes mellitus tipo II, obesidad e hipercolesterolemia). (Wang et al., 2020). Destacan también con la disbiosis las enfermedades alérgicas, enfermedades neurodegenerativas. (Bunyavanich \& Berin, 2019), y también en la enfermedad renal crónica, entre muchas otras más. (Cigarran Guldris et al., 2017)

Como se ha podido apreciar la inseguridad alimentaria comprende varias dimensiones donde confluyen, la falta de acceso a los alimentos por diferentes causas, en cantidad y calidad adecuada, que afecta mayoritariamente a la población de países en desarrollo, dentro de ellos existen grupos poblacionales más vulnerables, destacándose los niños, mujeres embarazadas, población indígena y personas de bajos ingresos económicos. (Assunta B et al., 2016), (Aulestia-Guerrero \& Capa-Mora, 2020)

\section{CONCLUSIONES}

Los impactos de la acción del hombre sobre el medio ambiente han modificado la disponibilidad de alimentos tanto en cantidad, como en la calidad, siendo este último atributo responsable de serios problemas de salud, pudiéndose señalar de manera significativa los daños al sistema cardiovascular, renal, endocrino, inmunológico y sistema nervioso. Urge una atención diferenciada a los programas de alimentación por parte de los gobiernos, que sean sostenibles y de acceso universal.

\section{REFERENCIAS BIBLIOGRÁFICAS}

Aguilar-Estrada, A. E., Caamal-Cauich, I., Barrios-Puente, G., Ortiz-Rosales, M. Á., Aguilar-Estrada, A. E., Caamal-Cauich, I., Barrios-Puente, G., \& Ortiz-Rosales, M. Á. (2019). ¿Hambre en México? Una alternativa metodológica para medir seguridad alimentaria. Estudios sociales. Revista de alimentación contemporánea y desarrollo regional, 29(53). https://doi.org/10.24836/es.v29i53.625

Altier, N., Abreo, E., Altier, N., \& Abreo, E. (2020). Una sola salud: Consideraciones en el Año Internacional de la Salud Vegetal. Agrociencia (Uruguay), 24(SPE2). https://doi.org/10.31285/agro.24.422 
Assunta B, M., Souza G, L., Arruda Teo, C. R. P., \& Pozzagnol, M. (2016). Condiciones socioeconómicas e higiénico-sanitarias como dimensiones de la seguridad alimentaria y nutricional. Revista chilena de nutrición, 43(1), 62-67. https://doi.org/10.4067/S0717-75182016000100009

Aulestia-Guerrero, E. M., \& Capa-Mora, E. D. (2020). Una mirada hacia la inseguridad alimentaria sudamericana. Ciência \& Saúde Coletiva, 25, 2507-2517. https://doi.org/10.1590/1413-81232020257.27622018

Baldassarre, M. E., Di Mauro, A., Capozza, M., Rizzo, V., Schettini, F., Panza, R., \& Laforgia, N. (2019). Dysbiosis and Prematurity: Is There a Role for Probiotics? Nutrients, 11(6), E1273. https://doi.org/10.3390/nu11061273

Borrego, P., \& Enrique, C. (2020). Cambio climático, inseguridad alimentaria y obesidad infantil. Revista Cubana de Salud Pública, 45, e1964.

Bunyavanich, S., \& Berin, M. C. (2019). Food allergy and the microbiome: Current understandings and future directions. The Journal of Allergy and Clinical Immunology, 144(6), 1468-1477. https://doi.org/10.1016/j.jaci.2019.10.019

Butel, M.-J., Waligora-Dupriet, A.-J., \& Wydau-Dematteis, S. (2018). The developing gut microbiota and its consequences for health. Journal of Developmental Origins of Health and Disease, 9(6), 590-597. https://doi.org/10.1017/S2040174418000119

Castañeda Guillot, C. (2018). Microbiota intestinal y salud infantil. Revista Cubana de Pediatría, 90(1), 94-110.

Cigarran Guldris, S., González Parra, E., \& Cases Amenós, A. (2017). Gut microbiota in chronic kidney disease. Nefrologia: Publicacion Oficial De La Sociedad Espanola Nefrologia, 37(1), 9-19. https://doi.org/10.1016/j.nefro.2016.05.008

Fernandez, A., \& Paoletti, C. (2018). Unintended Effects in Genetically Modified Food/Feed Safety: A Way Forward. Trends in Biotechnology, 36(9), 872-875. https://doi.org/10.1016/j.tibtech.2018.03.005

Liu, X., Steele, J. C., \& Meng, X.-Z. (2017). Usage, residue, and human health risk of antibiotics in Chinese aquaculture: A review. Environmental Pollution (Barking, Essex: 1987), 223, 161-169. https://doi.org/10.1016/j.envpol.2017.01.003

Moura, L. T. R. de, Bedor, C. N. G., Lopez, R. V. M., Santana, V. S., Rocha, T. M. B. da S. da, Wünsch Filho, V., \& Curado, M. P. (2020). Occupational exposure to 
organophosphate pesticides and hematologic neoplasms: A systematic review. Revista Brasileira De Epidemiologia = Brazilian Journal of Epidemiology, 23, e200022. https://doi.org/10.1590/1980-549720200022

Ni, J., Wu, G. D., Albenberg, L., \& Tomov, V. T. (2017). Gut microbiota and IBD: Causation or correlation? Nature Reviews. Gastroenterology \& Hepatology, 14(10), 573-584. https://doi.org/10.1038/nrgastro.2017.88

Pérez-Tamayo, E. M., Aguirre-Henao, C. D., Echavarría-Rodríguez, I. C., TamayoCastro, J. A., Pérez-Tamayo, E. M., Aguirre-Henao, C. D., Echavarría-Rodríguez, I. C., \& Tamayo-Castro, J. A. (2020). Análisis de políticas públicas alimentarias y nutricionales colombianas: Aproximación a las posturas epistemológicas, teóricas y metodológicas en los años 2000-2015. Revista de la Facultad de Derecho y Ciencias Políticas, 50(132), 192-214. https://doi.org/10.18566/rfdcp.v50n132.a09

Ramos-Ulate, C. M., Pérez-Álvarez, S., Guerrero-Morales, S., Palacios-Monarrez, A., Ramos-Ulate, C. M., Pérez-Álvarez, S., Guerrero-Morales, S., \& PalaciosMonarrez, A. (2021). Biofertilización y nanotecnología en la alfalfa (Medicago sativa L.) como alternativas para un cultivo sustentable. Cultivos Tropicales, 42(2). $\quad$ http://scielo.sld.cu/scielo.php?script=sci_abstract\&pid=S0258$59362021000200010 \& \operatorname{lng}=$ es\&nrm=iso\&tlng=es

Rumiato, A. C., \& Monteiro, I. (2017). Contaminantes em alimentos e orientação nutricional: Reflexão teórica. Revista de Salud Pública, 19, 574-577. https://doi.org/10.15446/rsap.v19n4.41939

Thursby, E., \& Juge, N. (2017). Introduction to the human gut microbiota. The Biochemical Journal, 474(11), 1823-1836. https://doi.org/10.1042/BCJ20160510

Tofiño Rivera, A. P., Carbono Murgas, R. E., Melo Ríos, A. E., \& Merini, L. J. (2020). [Effect of glyphosate on microbiota, soil quality and biofortified bean crop in Codazzi, department of Cesar, Colombia]. Revista Argentina De Microbiologia, 52(1), 61-71. https://doi.org/10.1016/j.ram.2019.01.006

Wang, P.-X., Deng, X.-R., Zhang, C.-H., \& Yuan, H.-J. (2020). Gut microbiota and metabolic syndrome. Chinese Medical Journal, 133(7), 808-816. https://doi.org/10.1097/CM9.0000000000000696 
López Gamboa y Elpidio Pérez...

Weiss, G. A., \& Hennet, T. (2017). Mechanisms and consequences of intestinal dysbiosis.

Cellular and Molecular Life Sciences: CMLS, 74(16), 2959-2977. https://doi.org/10.1007/s00018-017-2509-x 